\title{
Development of Crushable Shock Absorption Structure for OMOTENASHI Semi-Hard Impact Probes
}

\author{
By Tetsuya YAmada, ${ }^{1)}$ Hideyuki TANNO, ${ }^{2)}$ and Tatsuaki Hashimoto ${ }^{1)}$ \\ 1)Institute of Space and Astronautical Science, JAXA, Sagamihara, Japan \\ ${ }^{2)}$ Kakuda Space Center, JAXA, Kakuda, Japan
}

(Received June 30th, 2017)

\begin{abstract}
The crushable structure realizes not only chuteless landing on the planetary bodies with atmosphere but also on airless ones by absorbing landing shock energy and protects the inner instrument modules against the landing shock within a prescribed deceleration level. The lunar semi-hard landing mission OMOTENASHI proposed by JAXA is selected for launch by NASA SLS in 2018. The present study shows state-of-the-art technology development of the crushable shock absorption structure together with a design example for the small lunar semi-hard impact surface probe of OMOTENASHI.
\end{abstract}

Key Words: Shock Absorption, Crushable Structure, OMOTENASHI, Lunar Exploration, SLS

\section{Nomenclature}

A : projected area in the deceleration direction

$a_{\max }:$ maximum deceleration

$h \quad$ : required thickness for deceleration

$m \quad$ : mass to be decelerated

$V \quad$ : impact velocity

$\sigma_{P} \quad:$ plateau stress

$\varepsilon_{1}, \quad$ : beginning / end strain of plateau region

\section{Introduction}

Recently, target bodies of the solar exploration have extended to distant heavenly bodies beyond the Mars, and even to more distant ones. Because the overall mission duration tends to be longer and longer in such high-energy missions, reliability requirements for the subsystems become severer. The compact probe for semi-hard impact landing is one of the key technologies to accomplish such challenging exploration missions under small weight budget restraints. One of the important issues concerned with the semi-hard landing of compact probes is secure functional reliability of the subsystem and impact shock absorption. The lunar semihard landing mission OMOTENASHI proposed by JAXA has been selected for launch by NASA SLS in 2018.1) (Fig. 1).

The crushable structure realizes not only chuteless landing on the planetary bodies with atmosphere but also on airless ones by absorbing landing shock energy and protects the inner instrument modules against the landing shock within a prescribed deceleration level. These versatile landing is advantageous for secure decent system in that pyrotechnique devices, ignition electronics, parachute-triggering sensors, and triggering-control logic circuits etc. are no longer required at landing. Mechanical characteristics, mainly stress vs. strain characteristics, have been investigated for various materials such as porous metals, carbon structures and resins. It is crucial to control the mechanical characteristics parametrically according to the mission requirements such as allowable maximum deceleration, instrument weight, etc. Authors have been studying basics characteristics of behavior of the crushable material at high speed impact by using the crushable ball ejected through the ballistic range (Fig. 2) ${ }^{2,3)}$ : Synchronized to the OMOTENASHI master schedule, the R\&D works need to be accelerated taking account of effects of material size, sand target, and canted impact behavior. In order to verify validity of the analysis and extracting other issues concerned with crushable material, the landing impact tests are being carried out by using the ballistic range in JAXA Kakuda Space Center by impacting a small test model with onboard measurement systems onto the target simulating lunar soil. Measured impact data are compared to the analytical prediction and discussed.

The shock absorption system of the OMOTENASHI surface probe is now under trade-off stage among the present crushable structure and the airbag system. The present study shows state-of-the-art technology development of the crushable shock absorption structure together with a design example for the small lunar semi-hard impact surface probe of OMOTENASHI.

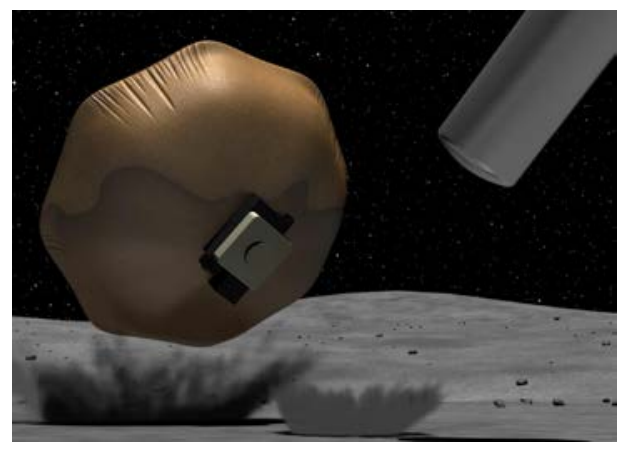

Fig. 1. Artist's impression of semi-hard landing of the compact surface probe for OMOTENASHI mission. After the deceleration delta-V by a small retro-motor, the probe impacts on the surface with the several tens $\mathrm{m} / \mathrm{s}$ correspondent to the residual velocity. 


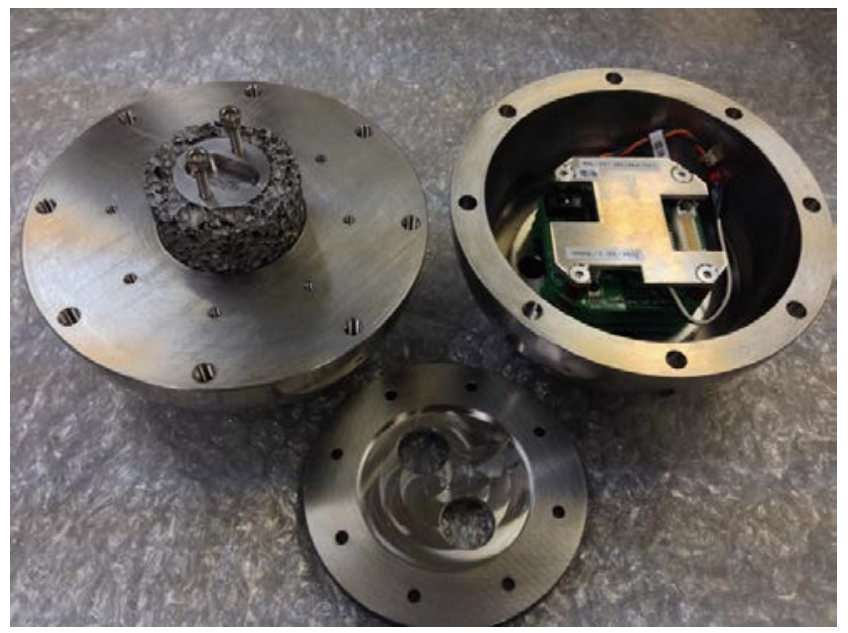

Fig. 2. The crushable-ball for the ballistic range impact test 2,3): An example of the crushable material is mounted in the center of the forebody shell (upper left in the photo). The data-logger is on the bottom of the aftbody shell (upper right).

\section{OMOTENASHI and Lunar Semi-Hard Landing Requirement}

After OMOTENASHI cube-sat mounted in the MSA (Stage Adapter) is launched by SLS EM-1 (Exploration Mission No. 1) rocket, it is separated and inserted into the lunar transfer orbit. Approaching to the perilunarapsis, the spacecraft ignites retro-solid motor for deceleration. At burnout, the relative velocity of the spacecraft and the moon surface should be minimized except the residual velocity due to several errors of thrust level, thrust direction, orbital determination etc. Though successive efforts are still under investigation to minimize the residual impact velocity in the fields of navigation optimization, orbit determination, attitude control schemes and solid motor development etc, the desirable nominal velocity $30 \mathrm{~m} / \mathrm{s}$ both in vertical and horizontal direction is not securely accomplished with large probability at the moment. ${ }^{4)}$ Thus the shock absorption subsystem has set extra preferable velocity requirement upto $50 \mathrm{~m} / \mathrm{s}$ so that the shock absorption would not insist extra severe requirements on the navigation system in the future as shown in Table 1.

Among devices of the onboard instruments, the quartz devices are considered to be weak against impact shock. Thus, the maximum quasi-static deceleration has been set to be 3,000G taking account of the successful landing experience of Hayabusa reentry capsule, in which the spaces between circuit boards are filled with comfort resin: Vibration tests for the onboard instruments are also undertaken in parallel.

\section{Crushable Material Consideration}

\subsection{Principle of crushable shock absorption}

The crushable material with constant plateau stress for certain ranges of the plateau strain is advantageous to restrict effectively the consequent deceleration rate within a designed value, which has been investigated and tested. In order to have foresight in the further design process, crushable material has been modeled as shown in Fig. 3. Applying the deceleration level $a_{\max }$ acceptable to the onboard instrument, the maximum plateau stress $\sigma_{p}$ is uniquely determined to be

$$
\sigma_{P}<\sigma_{P, \text { max }}=\frac{m}{A} a_{\text {max }},
$$

where $\mathrm{m}$ is summation of the instrument mass and crushable structure mass, and $A$ is the bottom projected area. As will be described in the following section, $\sigma_{p}$ in Eq. (1) is safe-side approximation at its maximum value because the squashed crushable material during the process of energy absorption does no longer behave as moving inertia mass, which acts to reduce the instantaneous deceleration compared with the

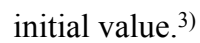

\section{Volumetric Energy Absorption}

When the energy is absorbed only in the plateau region and the amount absorbed in the elastic region is negligible, the following approximation is available :

$$
\frac{1}{2} m_{0} v_{0}{ }^{2}=V_{0} \int \sigma d \varepsilon \approx \sigma_{P} \varepsilon_{2} V_{0} .
$$

In order to absorb the total shock energy exclusively in the plateau region, the allowable initial material volume $V_{0}$ at minimum $V_{0, \min }$ is expressed as

$$
V_{0}>V_{0, \text { min }}=m_{0} v_{0}^{2} / 2 \sigma_{p} \varepsilon_{2} .
$$

The larger $\varepsilon_{2}$ is desirable for minimizing the material volume $\mathrm{V}_{0}$ at a given terminal velocity and plateau stress.

The minimum plateau-end-strain $\varepsilon_{2}$ is also determined under the condition that the landing shock energy is completely absorbed within the plateau region:

$$
\varepsilon_{2}>\varepsilon_{2, \min }=m_{0} v_{0}^{2} / 2 \sigma_{p} V_{0}
$$

\section{Thickness of the crushable material}

Because the constant deceleration is most effective, the minimum deceleration length $L_{\min }$ is uniquely determined as a

Table 1. Landing Velocity Requirement.

\begin{tabular}{lll}
\hline & Nominal & Design Preferable \\
\hline Vertical Velocity & $\sim 30 \mathrm{~m} / \mathrm{s}$ & $\sim 50 \mathrm{~m} / \mathrm{s}$ \\
Horizontal Velocity & $\sim 30 \mathrm{~m} / \mathrm{s}$ & $\sim 50 \mathrm{~m} / \mathrm{s}$ \\
\hline
\end{tabular}

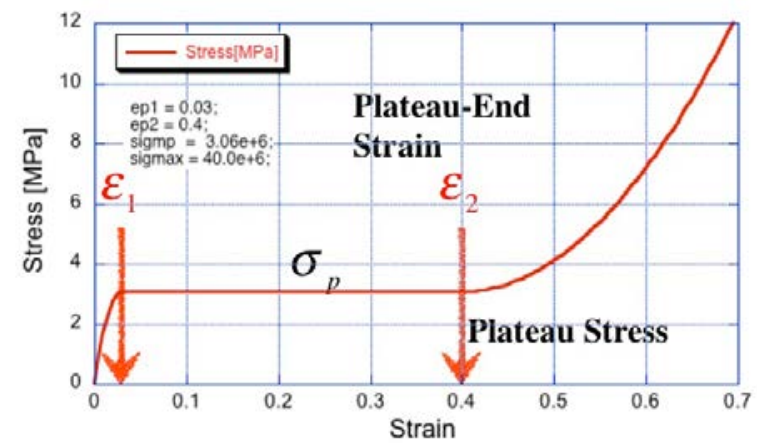

Fig. 3. Modeling of the stress vs. strain characteristics of the crushable material. 
function of the impact velocity and the deceleration limitation (in case that the no other energy dissipation is negligible) as expressed in

$$
L_{\min }=\frac{V^{2}}{2 a_{\max }} .
$$

$L_{\min }$ equals $15.3 \mathrm{~mm}$ when $\mathrm{V}=30 \mathrm{~m} / \mathrm{s}$ and $a_{\max }=3000 \mathrm{G}$. The lager plateau end value is desirable for minimizing the thickness of the material as shown in

$$
h=\frac{L_{\min }}{\varepsilon_{2}}=\frac{V^{2}}{2 \varepsilon_{2} a_{\max }} .
$$

Assuming $\varepsilon_{2}$ be $60 \%$ as shown in the later section, the minimum thickness of the material is $25.5 \mathrm{~mm}$. The remarkable point is that the minimum thickness of the material is uniquely determined by the impact velocity, allowable max. deceleration and plateau end strain. Thus, the larger plateau-end strain is desirable for minimizing the thickness for effective shock energy absorption.

\subsection{Design and requirements for crushable material}

Requirements for the crushable material to be satisfied in the present designing are summarized as follows;

1)to have appropriate transfer function of such that suppresses the shock response spectrum be within subsystems' requirements.

2)transformation is negligible against the static load during the long orbital flight.

3)performance degradation and transformation is negligible in the vacuum, temperature and radiation condition.

4)made of material free from outgassing and solid particle scattering.

5)radio-wave transparent material.

6)Isotropic

Though the cellular porous metal was one of the promising candidate for the crushable material satisfying requirements listed above except 5), we have newly selected a kind of urethane material as a primary candidate because the requirement 5) radio-wave transparency is especially important in the present small probe mission. Based on the commercially manufactured material, a new version of the urethane material has been developed so that the characteristics satisfy the appropriate plateau stress as shown in Fig. 4. The plateau stress is designable by controlling the

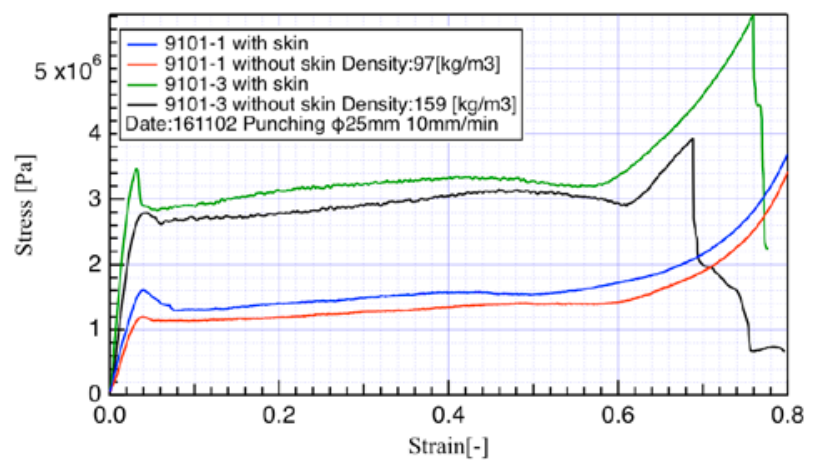

(A) Stress-Strain Characteristics of the shock absorption material.

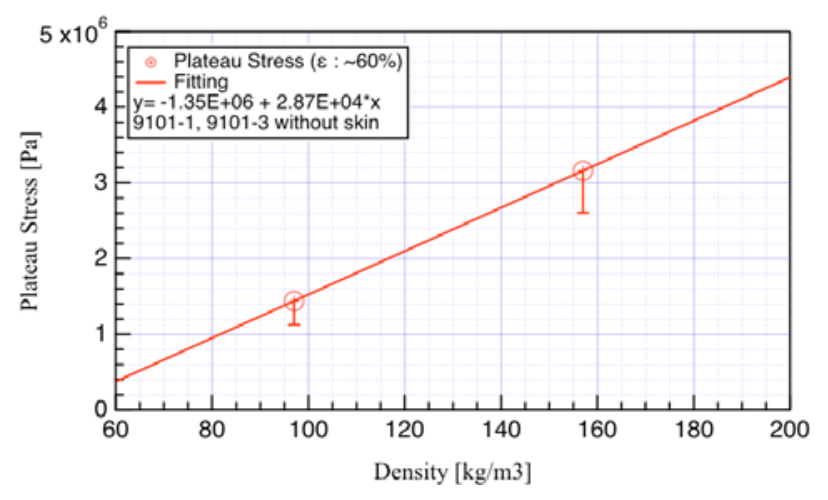

(B) Plateau Stress as a function of Density

Fig. 4. Stress-Strain Characteristics of the OMOTENASHI shock absorption material.
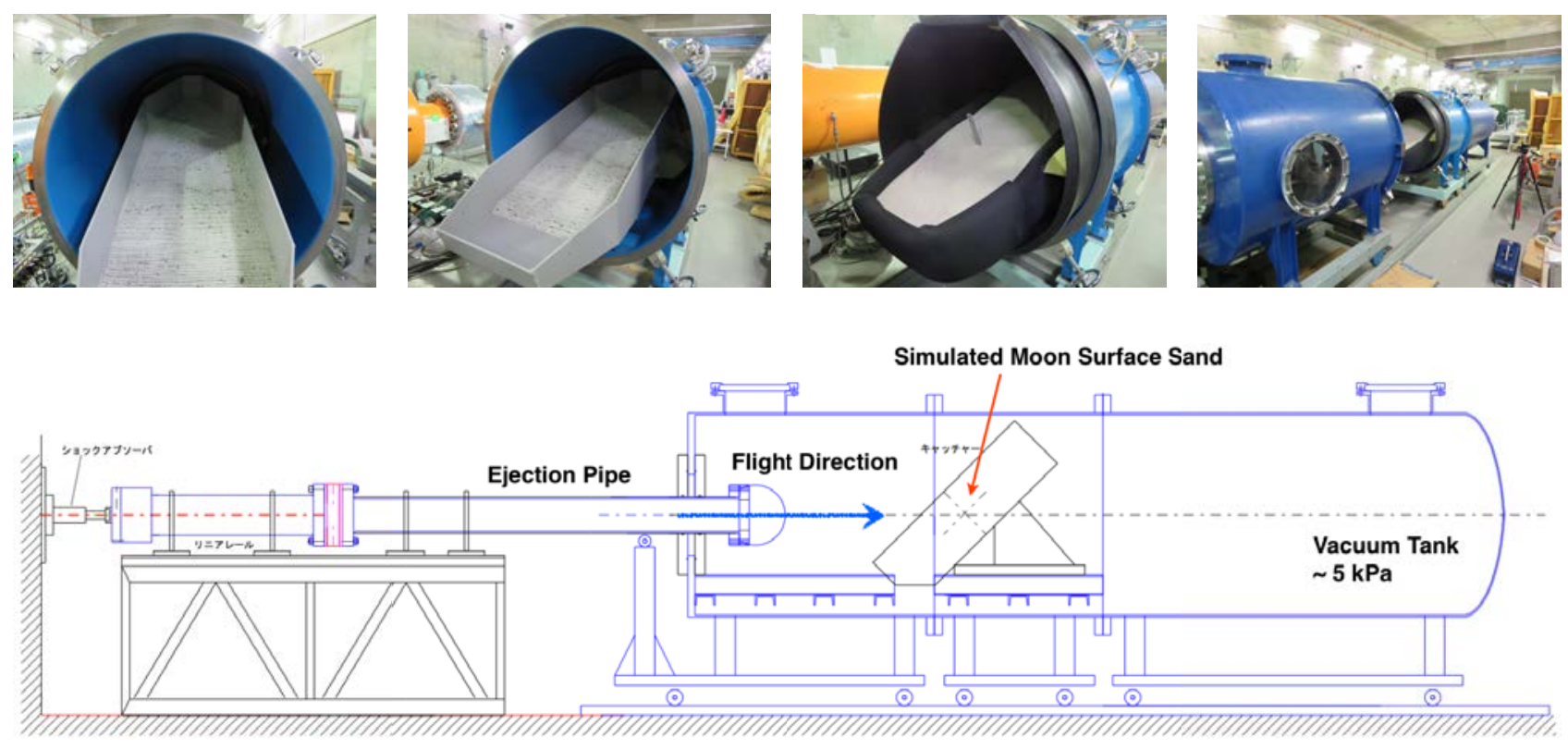

Fig. 5. The ballistic range configuration for the OMOTENASHI simulated lunar soil impact tests. 
density. The candidate urethane material have exhibited excellent plateau till $60 \%$ end-strain.

Though it does not exhibit drastic difference in quasi-static compression test at the order of several $\mathrm{cm}$ per minute, the strain-stress characteristic seem to depend on the deformation strain-rate especially in the present high speed deformation. In the preliminary analysis of the data : The material may be stiffer in the high speed deformation, which is planned to be investigated later after parametrically carried-out experiments.

\subsection{Simulated lunar sand impact test}

It is of great interest whether the small probe enters into deep in the lunar soil at impact or not, which will lead to communication failures even after successful landing. Behavior of the sand in the atmospheric environment is different from that in the vacuum condition, because the air included in-between sands helps propagation of the impact force and much sand is to be scattered at landing in the atmosphere. Impact tests in the vacuum condition are safer from perspective that we need to evaluate the possibility of diving into the soil. Thus the vacuum tank has been equipped to the conventional impact test configuration as have been used. ${ }^{\text {) }}$

Experiment configuration of the ballistic range in Kakuda Space Center is shown in Fig. 5. The test models are attached to the sabot and inserted into $\phi 160 \mathrm{~mm}$ pipe connected to the vacuum tank. By bursty break of the partition film between high-pressure region and the vacuum, the test model is ejected into the vacuum tank with prescribed velocity according to the thickness of the partition film made of Myler ${ }^{\mathrm{TM}}$.

As simulated lunar surface sands, we have adapted silica sands no. 5, which is, in general, made of over $95 \%$ of $\mathrm{SiO}_{2}$ with minor impurities of $\mathrm{Al}_{2} \mathrm{O}_{3}, \mathrm{Fe}_{2} \mathrm{O}_{3}, \mathrm{Na}_{2} \mathrm{O}$, etc. Over $80 \%$ of particle size are distributed mainly between 300 to 600 $\mu \mathrm{m}$. The silica sands no. 5 is considered to be appropriate as simulated lunar soil from the standpoint of surface friction which may causes intense rotation up-to unpredicted rate at landing.

The measurement system for the impact shock deceleration consists of the 3-axis accelerometer and the data logger connected to the lithium-polymer battery, specifications of which are summarized in Table 2. The measurement systems is accommodated and covered with rectangular aluminum case (Outer shape : $74 \times 60 \times 28$ ), which means the impact deceleration is different in the impact direction and projected area of the instrument case by 2.6 times $((78 \times 60) /(60 \times 28))$ at maximum when the uniform material is used for shock absorption. Though the material characteristics needs to be selected for each direction and projected area of instrument case for the flight model, the experiment model have been designed with the averaged value of 4,000G.

\section{Results and Discussion}

\subsection{Possibility of diving into soil}

Figure 6 shows typical photos of the poly-ethylene projectile impacted on the $23^{\circ}$ canted target soil taken by 2,000 fps high-speed camera. Though the impact angle are not parametrically varied due to limitations in the experiment configuration, the tendency of diving deep into the soil was not observed up-to $100 \mathrm{~m} / \mathrm{s}$ impact velocity: the sands were scattered widely withe several msec of time-lag from the impact. The possibility of diving into sands is thought to be small from these tests. The rotation rate caused at impact was estimated to be an order of 1 revolution per $10 \mathrm{msec}$, which leads to centrifugal force of about $300 \mathrm{G}$. The translational deceleration is thought to be dominant even at shallow angle impact as shown in the later section.

\subsection{Survivability of the probe at impact}

Several impact tests have been carried out with several impact velocity levels. Figure 7 shows typical high-speed camera photos. The original urethane test model was broken at impact as shown in Fig. 7 (A). In general the material with plateau stress have brittle property and is weak in the tensile

Table 2. Accelerometer and Data Logger Specifications.
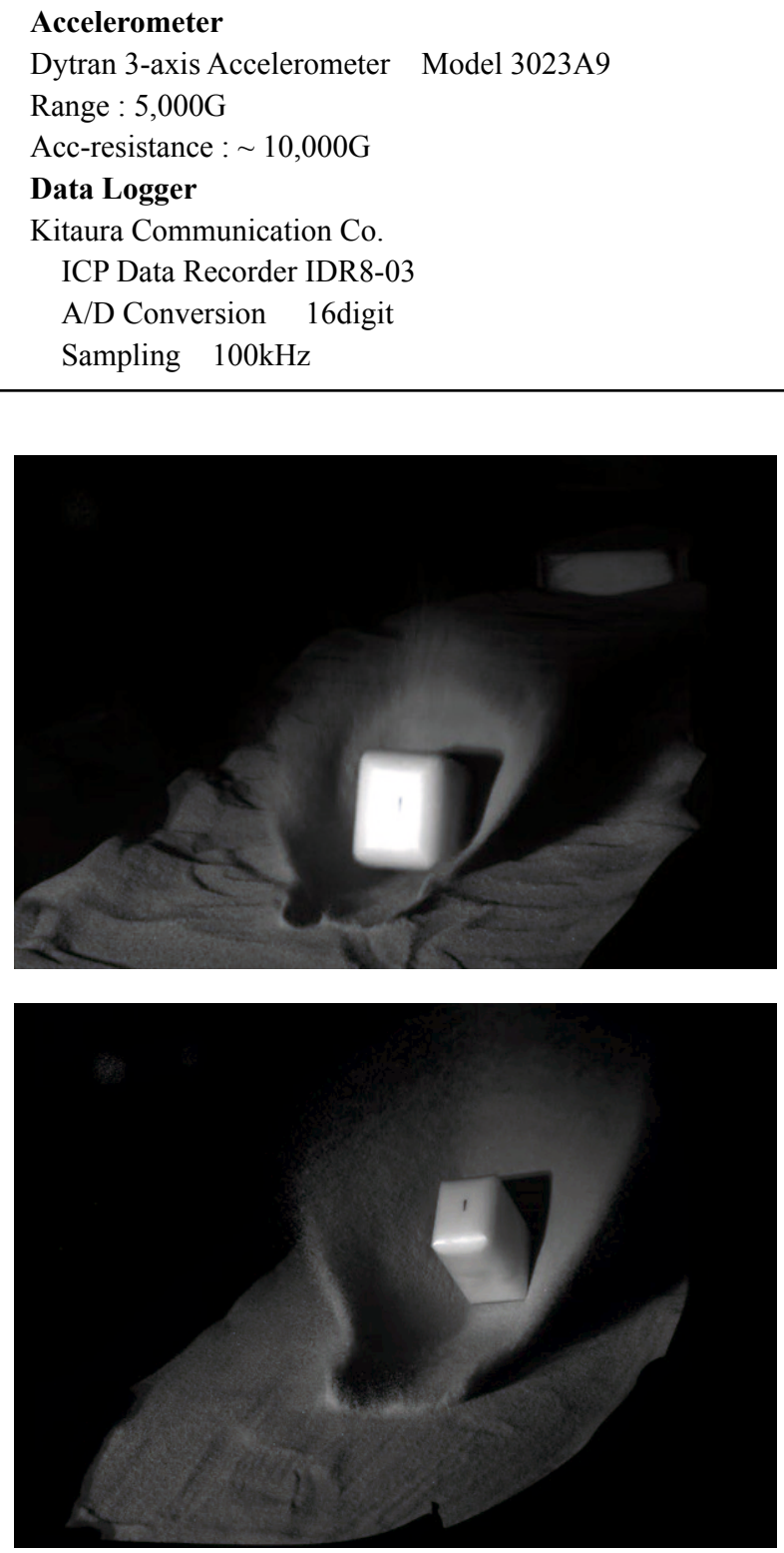

Fig. 6. Poly-ethylene test model for preliminary impact test. (Target : $23^{\circ}$ simulated lunar sand soil, Velocity : $37 \mathrm{~m} / \mathrm{s}$ ) 
(A) $40 \mathrm{~m} / \mathrm{s} 20^{\circ}$ Impact (w/o Surface Fibre-reinforced Tape ).
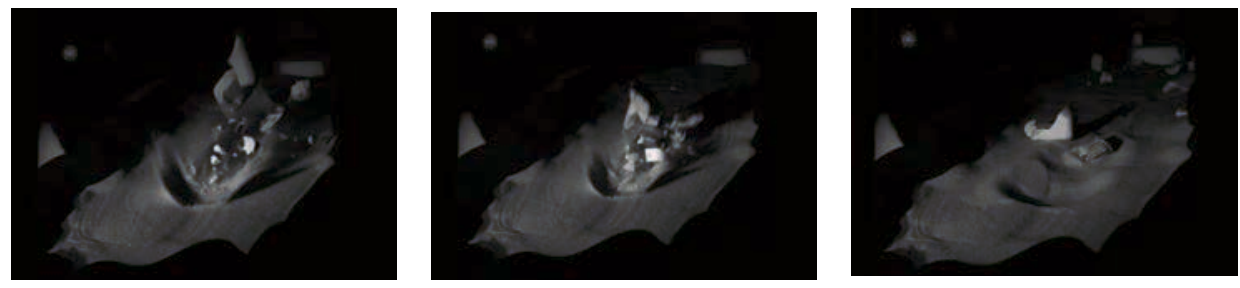

(B) $40 \mathrm{~m} / \mathrm{s} 20^{\circ}$ Impact (w/ Surface Fibre-reinforced Tape ).
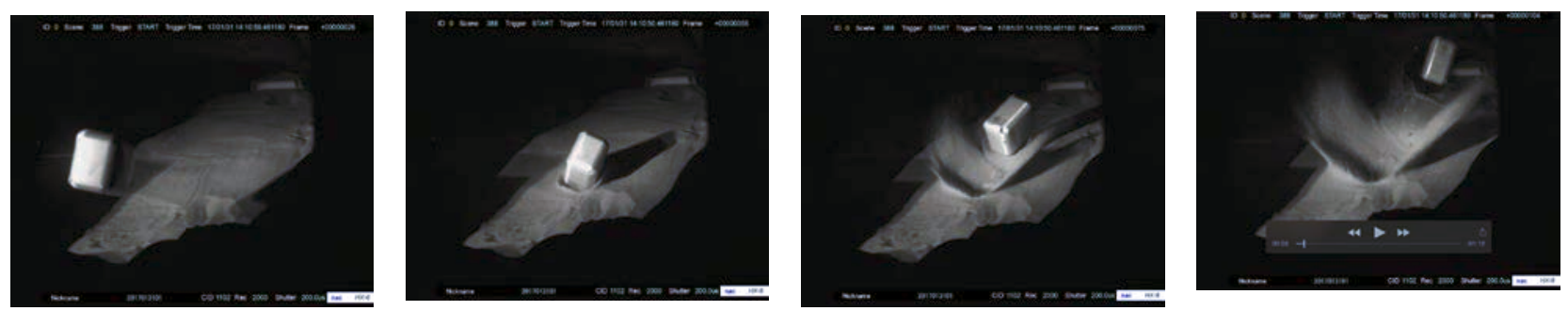

(C) $110 \mathrm{~m} / \mathrm{s} 20^{\circ}$ Impact (w/ Surface Fibre-reinforced Tape ).
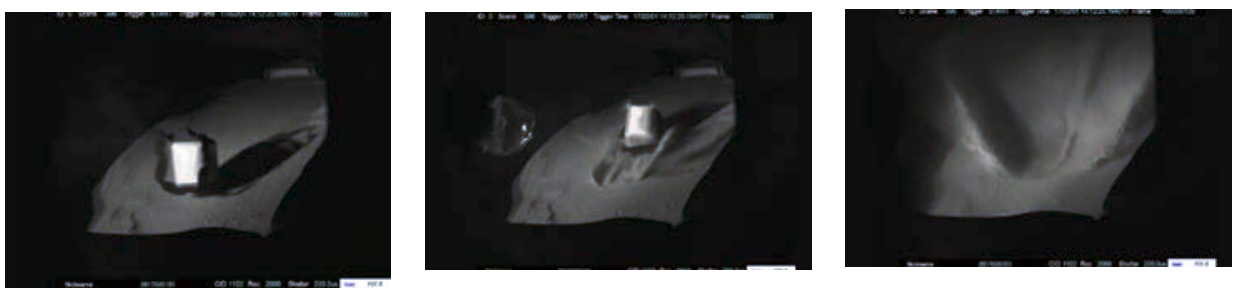

Fig. 7. Urethane crushable material tests with various impact velocity.

stress and shear stress. The tensile and shear strength of the present urethane material is thought to yield to the stress converted from the compressive stress as a consequence of Poisson-expansion to the direction different from the impact direction, though the exact attitude angle of the test model is not determined.

It has been demonstrated that the break-up of the material described above can be avoided merely by covering the surface with glass-fiber tape with thickness of about $300 \mu \mathrm{m}$ as shown in Fig. 7(B). The tensile stress and shear stress is thought to be distributed properly to the glass-tape. The qualitative measurement of the tensile stress of the tape are required soon, the urethane test model has successfully survived $110 \mathrm{~m} / \mathrm{s}$ velocity test on the $23^{\circ}$ canted soil as shown in Fig. 7(C).

\subsection{Energy balance at impact on sands}

Typical cross-section of the urethane test model after impact test on $23^{\circ}$-canted simulated lunar soil with velocity of $37 \mathrm{~m} / \mathrm{s}$ was shown in Fig. 8 . About $10 \mathrm{~mm}$ thickness is measured to be compressed among total $25 \mathrm{~mm}$ thickness.

Though the attitude and exact impact direction of the test model is not determined, the total kinetic energy $\mathrm{E}_{0}$ based on the normal velocity and the energy $E_{1}$ based on the velocity component vertical to the sand surface is estimated to be

$$
E_{0}=\frac{1}{2} m V^{2}=150.6[\mathrm{~J}]
$$

and
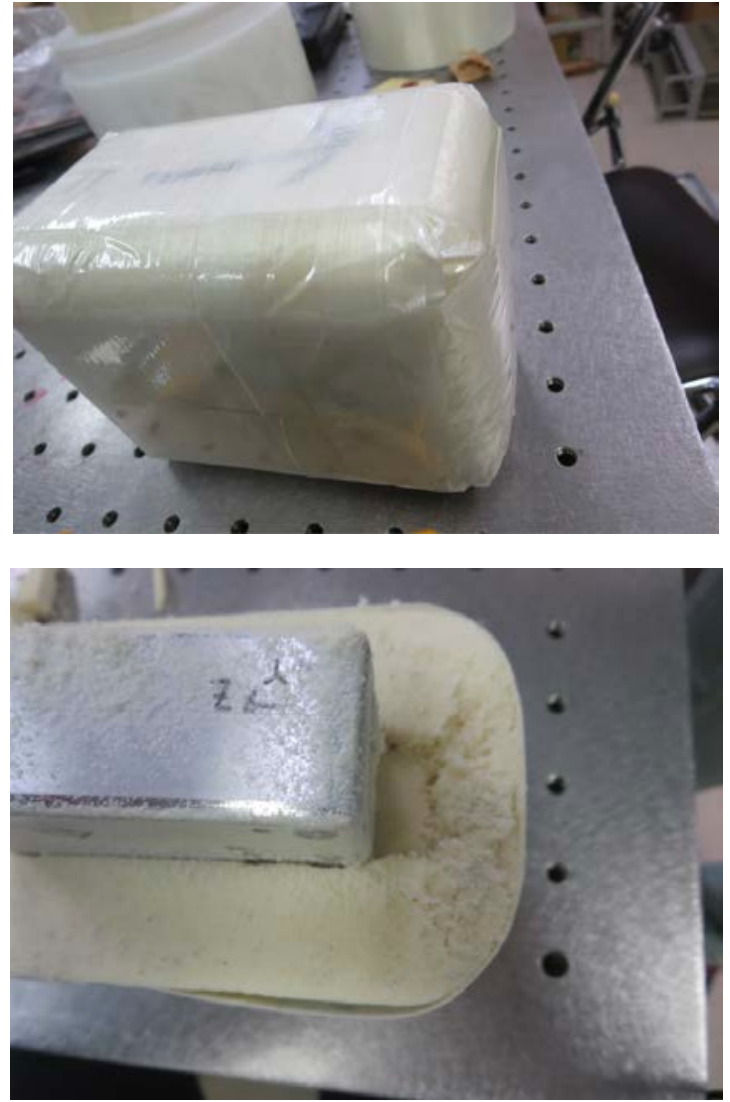

Fig. 8. Typical Cross-section of the Urethane Test Model after Impact (Target : $23^{\circ}$ simulated lunar sand soil, Velocity : $37 \mathrm{~m} / \mathrm{s}$ ): About $10 \mathrm{~mm}$ thickness is measured to be compressed among total 25 mm thickness. 
Table 3. Averaged Deceleration predicted in each direction (inst. mass : $220 \mathrm{~g}$, ave. plateau stress : $1.3 \mathrm{MPa}$ ).

\begin{tabular}{cccccc}
\hline & $\begin{array}{c}\text { Area } \\
{\left[\mathrm{mm}^{2}\right]}\end{array}$ & $\begin{array}{c}\text { Prediction } \\
{[\mathrm{G}]}\end{array}$ & $\begin{array}{c}\text { Measured } \\
\text { Max [G] }\end{array}$ & $\begin{array}{c}\text { Meas./ } \\
\text { Predict }\end{array}$ & Angle \\
\hline $\mathrm{X}$ & 4440 & $2.68 \times 10^{3}$ & $1.5 \times 10^{3}$ & 0.56 & $56^{\circ}$ \\
$\mathrm{Y}$ & 2072 & $1.25 \times 10^{3}$ & $1.1 \times 10^{3}$ & 0.88 & $28^{\circ}$ \\
$\mathrm{Z}$ & 1680 & $1.01 \times 10^{3}$ & $0.3 \times 10^{3}$ & 0.3 & $72^{\circ}$ \\
\hline
\end{tabular}

$$
E_{1}=\frac{1}{2} m V \sin \alpha=23[J],
$$

where m:instrument mass $220 \mathrm{~g}$, V: impact velocity $37 \mathrm{~m} / \mathrm{s}$, and $\alpha$ : impact angle $23^{\circ}$. While the strain energy $\mathrm{E}_{2}$ used for the compression is

$$
E_{2}=\sigma_{p} A \cdot L=21.8[J]
$$

where $\sigma_{p}$ : the plateau stress $1.3 \mathrm{MPa}, \mathrm{A}$ : area $1680 \mathrm{~mm}$, and $\mathrm{L}$ : compressed distance $10 \mathrm{~mm}$. The kinetic energy of the onboard instrument absorbed by the crushable material is thought to be between vertical component and total energy: especially contribution of the horizontal component is not so large and of the same order of the vertical.

Though the fraction of the impact energy absorbed by the sand would differ according to the impact velocity and angle, the attitude of the model, and conditions of the sands etc, it is remarkable that not a small amount of the energy can be absorbed by the simulated lunar soil, which is very advantageous if the landing lunar surface conditions can be selected to the sand area without rocks. Unfortunately such precise attitude control of the surface probe can not be planned in the present mission. The energy absorption by surface soil should be considered to be optional from mission safety reason.

\subsection{Impact shock measurement}

Time-profile of the deceleration has been measured by onboard measurement system as shown in Fig. 9. The impact phenomenon is completed within $2 \mathrm{msec}$ after impact, which is almost doubled compared to the prediction, which is correspondent to the impact on the absolute rigid target such as concrete block.

The maximum deceleration differs in the direction according to the projected area, and it can be calculated based on Eq. (1) at constant plateau stress of $1.3 \mathrm{MPa}$ as shown in Table 3. The predicted deceleration value has been measured in each direction. The quotient of the measured value divided by the prediction is also shown in the table, which is identical to the direction cosine of the deceleration vector with regard to each base vector $\mathrm{X}, \mathrm{Y}, \mathrm{Z}$. Though the attitude at impact observed from the high-speed video is almost identical to the attitude estimated from the direction cosine.

As was previously shown in the cross-section in Fig. 8, the crushable material itself is broken and still maintains its shape due to support of the surrounding fiber reinforced tape. The

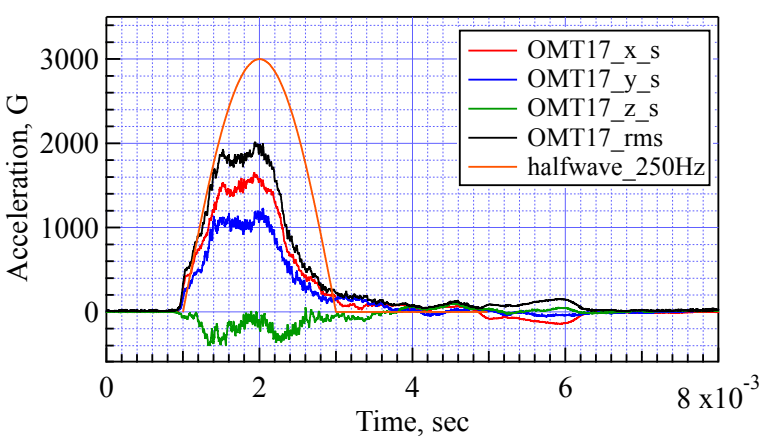

Fig. 9. Measured 3-axis Deceleration Profile with low-density urethane shock absorption material (OMT-17 Target : $23^{\circ}$ simulated lunar sand soil, Velocity : $37 \mathrm{~m} / \mathrm{s}$ ) with the half-sine wave profile of $250 \mathrm{~Hz}$ superimposed over as a reference.

energy dissipated to the break-up process of the material or sand soil should not act on decreasing the averaged deceleration value. The impact duration against absolute rigid targets such as concrete block was measured to be about 1 msec. ${ }^{3)}$ The enlarged impact time compared to the rigid targets may be main cause of the lower deceleration than predicted.

\subsection{Shock response spectrum analysis}

Figure 10 shows an example of SRS (shock response spectrum) analysis of the deceleration profile shown in Fig. 9. Compared with the SRS of the half-sine wave with frequency of $250 \mathrm{~Hz}$, the norm of the deceleration exceed it in the frequency range blow $50 \mathrm{~Hz}$, which is considered to be caused by the measurement offset drift around zero-G and is not essential to the spectrum analysis. The peak acceleration up to about $3,000 \mathrm{G}$ is estimated around frequency from 400 to 500 $\mathrm{Hz}$, which requires careful design or treatment for the onboard instrument because the resonant frequency of the instruments tend to lie in the frequency region. Further SRS analyses are planned because it is important for determining the mechanical environment for onboard instrument of the surface probe at impact. It is future issue whether the amplitude of SRS at a given higher frequency can be relaxed enough by increasing energy dissipation in the material with lower density.

\section{Conclusion}

State-of-the-art technology development of the crushable shock absorption structure together with a design example for the small lunar semi-hard impact surface probe of OMOTENASHI. Though within limited experiment conditions of impact tests on the canted simulated lunar surface soil target, the possibility of diving into sands is thought to be small. And the rotational centrifugal force caused at impact has turned out not to be dominant compared to the translational shock deceleration. The break-up of the brittle crushable material at impact can be avoided by covering the surface with glass-fiber tape with thickness of about $300 \mu \mathrm{m}$. Though qualitative measurements of the tensile stress of the tape are required soon, the urethane test model has successfully survived $110 \mathrm{~m} / \mathrm{s}$ velocity test on the $23^{\circ}$ canted soil due to remarkable that not a small amount of the 


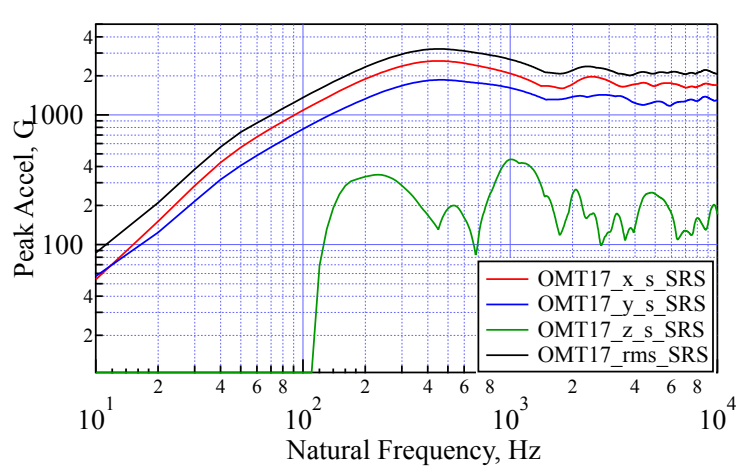

Fig. 10. Shock Response Spectrum analysis example (OMT-17 Target : $23^{\circ}$ simulated lunar sand soil, Velocity : $37 \mathrm{~m} / \mathrm{s}$ ).

landing shock energy can be absorbed by the simulated lunar soil. The fact is very advantageous if the landing lunar surface conditions can be selected to the sand area without rocks.

\section{Acknowledgement}

The authors are deeply grateful to graduate student $\mathrm{Mr}$. Nakamura for their support and understanding in the series of tests. And also, the authors debt is to Prof. Kitazono in Tokyo Metropolitan University, who gave insightful comments and suggestions on the crushable material.

\section{References}

1) Hashimoto, T., Yamada, T., Kikuchi, J., Otsuki, M., and Ikenaga, T. : Nano Moon Lander: OMOTENASHI, 31st International Symposium on Space Technology and Science, Matsuyama, ISTS-2017-f-053, 2017.

2) Yamada, T., Ogasawara, Kitazono, K., and Tanno, H. : HighSpeed Compact Entry Capsule Enhanced by Lightweight Ablator and Crushable Structure, Trans. JSASS Aerospace Tech. Japan, 14, ists30(2016), pp.Pe_33-Pe_40.

3) Nakamura, K., Yamada, T., Tan'no H., and Kitazono, K. : Analytical Study of Landing Shock Absorber in the High-Speed Impact Tests, 31st International Symposium on Space Technology and Science, Matsuyama, ISTS-2017-c-055, 2017.

4) Ozawa, Y., Takahashi, S., Hernando-Ayuso, J., Campagnola, S., Ike-naga, T., Yamaguchi, T. and Sarli, B. : OMOTENASHI Trajectory Analysis and Design: Earth-Moon Transfer Phase, 31 st International Symposium on Space Technology and Science, Matsuyama, ISTS-2017-f-054, 2017.

5) Tanno, H., Komuro, T., and Ito, K. : Free-Flight Aerodynamic Test with Projectile-Onboard Recorder in a Ballistic Range, AIAA Paper 2013-0475, 2013.

6) Gibson, L. J. and Ashby, M. F. : Cellular Solids Structure and Properties, Cambridge Univ. Press, 1997.

7) Tada, R. and Kitazono, K. : Effect of Strain Rate on the Compression, Indentation and Punching Deformation Behaviors of Porous Aluminum, The Japan Society of Mechanical Engineers M\&P 2015(23), pp. 322-1 - 322-5.

8) Kobashi, M. : Current Situation and Future Prospects of Porous Metals, Journal of Japan Institute of Light Metals, 55(2005), pp. 327-332.

9) Yamada, T., Tanno, H., and Kitazono, K.: Study on Crushable Structures for Landing Shock Relaxation of Small Capsules, 7th JSASS Space Science \& Tech. Lecture Meeting, 2H15, 2014.

10) Duffy, J. : The Dynamic Plastic Deformation of Metals: A Review, Report No. AFWAL-TR-82-4024, Materials Laboratory, Air Force Wright Aero-Nautical, Laboratories, Wright-Patterson Air Force Base, 1982, pp.11-19. 